\title{
ZU BESONDERHEITEN DES ÖSTERREICHISCHEN AUS SICHT EINES AUSLANDSGERMANISTEN
}

\author{
ANDRZEJ LEWANDOWSKI \\ Universitat Gdañsk
}

ABSTRACT. The main aim of the article is creating a basis for application of the most typical vocabulary for the area of Austria in teaching materials for German students. In the article there is a short analysis of the most common words of Austrian origin in everyday life, their etymology, peculiarity and typical lexical and phonetic differences between German and Austrian. The analyzed examples were taken from Austrian press and popular Austrian dictionaries.

Der vorliegende Artikel und eine genauere Analyse der Austriazismen in meiner geplanten Doktorarbeit sollen die Grundlagen dafür schaffen, damit die meist für Österreich typischen Wörter in den glottodidaktischen Materialien für die Germanistikstudenten verwendet werden können.

$\mathrm{Zu}$ der sprachlichen Sonderstellung Österreichs haben viele geschichtliche Aspekte beigetragen, u.a. Ablehnung der Konfession und damit der neuen Schriftsprache.

In Österreich haben sich die sprachlichen Eigenheiten stärker ausgeprägt als in den Sprachlandschaften innerhalb der Bundesrepublik Deutschland. Obwohl Deutsch hier als Amtssprache gilt, ist Österreich ein selbständiger Staat, der auf einer langen Tradition aufbaut. Im größten Teil gehört Österreich zu dem bairischen Dialektraum', nur in dem Bundesland Vorarlberg wird alemannisch

1 „Dialekt, Mundart: meist verstanden als örtlich gebundene, natürliche und im Alltag gebrauchliche Rede oder als besondere Ausdrucksweise der Sprachgemeinschaft eines Ortes oder einer Gegend, als ortlich bedingte sprachliche Sonderform vor dem Hintergnund einer ubberregionalen Standardsprache, die hinsichtlich des Gebrauchs sich zu dieser komplementâr verhallt. Aus historisch-genetischer Sicht erscheint die Hoch- oder Gemeinsprache als hervorgegangen bzw. herausentwickelt aus einem bestimmten Dialekt, denn wenn man die Sprache sich selbst uberläßt so kennt sie nur Dialekte, von denen keiner die Oberhand uber die anderen gewinnt (de Saussure 1967:234). Die Ursachen der raumlich-dialektalen Differenzierung liegen auch nach de Saussure in der historischen Veränderung der Sprachen, d.h. in ihrer mangelnden Beständigkeit, denn Entfernung allein schaffe keine Verschiedenheit. Jede Neuerung bestehe aus Einzelvorgängen lautlicher, lexikalischer, morphologischer und syntaktischer Art. Der extremen Aufspaltung in Dialekten wirken extralinguistische Faktoren entgegen: Handel und Verkehr, kulturelle u.a. Formen der Kommunikation" (Lewandowski 1990:220). 
gesprochen. Die Tatsache, daß sich, abgesehen von den Mundarten und der Umgangssprache, die deutsche Hochsprache in Österreich in mannigfacher Hinsicht von der sogenannten deutschen Gemeinsprache und deren besonderen Ausprägungen in den verschiedenen anderen Teilbereichen des deutschen Sprachraumes unterscheidet, ist den Fachleuten bekannt, fällt aber auch den meisten Sprachbenutzern auf. Abweichungen vom Binnendeutschen, d.h. dem Deutsch, das innerhalb der Grenzen des ehemaligen Deutschen Reiches gesprochen wird, finden wir in Syntax, Flexion, in der Aussprache, vor allem aber im Wortschatz. In dem vorliegenden Artikel handelt es sich vorwiegend um Ausdrücke, die im Bereich des praktischen Lebens verwendet werden, sowohl um die Standard- als auch Nonstandardvarietäten (vgl. Ammon 1995:3).

$\mathrm{Zu}$ den sogenannten bairisch-österreichischen Kennwörtern gehören u.a. Semmel für Brötchen, Kren für Meerrettich, oder Krapfen für Berliner Pfannkuchen.

Trotz vielfach andersartiger Wortbestände hat aber auch Vorarlberg an jenem spezifisch österreichischen Wortschatz teil, der sich durch die alte politische Sonderstellung am Rande des gemeindeutschen Sprachraumes entwickelt hat. Wörter wie Jause für eine Zwischenmahlzeit am Vor- und Nachmittag oder Korrespondenzkarte für Postkarte haben vor der Grenze nicht haltgemacht, werden aber z.B. im benachbarten Bayern nicht mehr verstanden. Der Ausdruck Jause, der nach P. Kretschmer schon im 15. Jahrhundert belegt ist, ist slowenischen Ursprungs; slow. júžina $=$ Mittagessen (Ammon 1995:180). Zwei Sachbereiche sind es vor allem, in denen die Besonderheiten des Ōsterreichischen Wortschatzes sinnfällig werden: die österreichische Amtssprache, die sich in der österreichisch-ungarischen Monarchie entwickelt hat und auch die österreichische Küchensprache, die jahrelang unter dem Einfluss der benachbarten Kulturen stand und in die viele Wörter aus den Nachbarsprachen gelangt sind.

\section{Zu Besonderheiten der Aussprache}

Das österreichische Deutsch unterscheidet sich in den Aussprachegewohnheiten vom Binnendeutschen, besonders im nord- und mitteldeutschen Raum, weist aber viel Gemeinsames mit Süddeutschland auf, vor allem mit Bayern. Die Konsonanten $b, d, g$ und $s$ werden in Österreich nicht stimmhaft, sondern stimmlos ausgesprochen, was nicht mit der Siebschen Normforderung übereinstimmt. Stimmhafte Konsonanten waren in Österreich nie bodenständig und kommen in der Hochsprache meistens angelernt vor. Je weiter man von der Hochsprache zur Umgangssprache geht, desto mehr wird der Aussprachegegensatz stimmhaft-stimmlos ersetzt durch stark-schwach (Lenis-Fortis), im mittelbairischen Dialekt fehlt schließlich der Unterschied ganz (Ebner 
1980:219). Das am Wortanfang stehende ch wird in den Fremdwörtern wie Chirurg, Chemie, China nicht als Reibelaut [x] oder [f], sondern als Verschlußlaut [k] ausgesprochen. Das geschriebene $a$ wird oft so wie ein [o] wie z.B. in Gasse oder wie ein [э] wie z.B. in Blase ausgesprochen.

Das lange schriftsprachliche $a \tilde{a}$ wird heute meistens nicht offen, sondern geschlossen wie ein langes $e$ ausgesprochen, so daß die folgenden Paare: $n a \overline{-}$ hen : drehen, Träger : Erreger, sie wären : sich wehren miteinander reimen.

Sowohl in Österreich als auch in Deutschland kommt es oft zur Vokalisierung der Endung [-er] zu einem a-artigen Vokal (Wiesinger 1988:23), z.B. Kinder, Vater oder Mutter. Die Endung [-ig], wie in Essig, füfzig, windig, lebendig wird nicht als [-ic] mit Reibelaut, sondern so wie es geschrieben wird mit dem Verschlußlaut [g] ausgesprochen, also anders als es in dem Siebschen Standardwerk geschrieben steht. $\mathrm{Zu}$ der für Ostösterreich charakteristischen Gewohnheit gehört die Erscheinung, daß schriftsprachliches $e i, a u$, eu etwa in Eis, Haus, Häuser nicht diphtongisch als ai, au, oü, sondern nach Wiener Vorbild monophtongisch als sehr offenes $a ̈, d, o ̈$ ausgesprochen werden.

$\mathrm{Zu}$ den Besonderheiten der Aussprache sind auch die Unterschiede (gegenüber dem Binnendeutschen) in der Betonung der Fremdwörter anzurechnen, z.B. Kaffée, Tabák, oder Mathemátik. Weitere wichtige Angaben dazu gibt es in den Arbeiten von Ulrich Ammon (1995) und Günter Lipold (in Wiesinger 1998).

\section{Gliederung des Wortschatzes}

Der im Österreichischen Deutsch schriftsprachlich gebräuchliche Wortschatz kann auf Grund seiner Verbreitung in folgende Gruppen gegliedert werden (vgl. Wiesinger 1988:25f.):

Süddeutscher Wortschatz:

z.B. Bub : Junge, Samstag: Sonnabend, heuer: dieses Jahr

Bairisch-österreichischer Wortschatz:

z.B. Scherzel : Endstück des Brotleibs, Kren: Meerrettich

Gesamtösterreichischer Wortschatz:

z.B. Marille : Aprikose, Jause : Zwischenmahlzeit am Vor-oder Nachmittag, $O$ bers : Sahne, sich verkuhlen : sich erkäten

Diese Gruppe umfasst auch politische und verwaltungstechnische Terminologie: z.B. Nationalrat : Bundestag, Parlament : Bundeshaus, Landeshauptmann : Ministerpräsident, Obmann : Vorsitzender (eines Vereines), Matura : Abitur, Erlagschein: Zahlkarte (bei der Post). 
Ostösterreichischer Wortschatz:

z.B. Fleischhauer, Fleischhacker : Metzger, Rauchfang : Kamin, Gelse : Mücke, Paradeiser: Tomate

Regionaler Wortschatz:

z.B. Sturm : gärender Traubensaft / in Garung ubergangener Most, Heuriger : vorübergehend eingerichtete Schankstatte des neuen Weins / junger Wein im ersten Jahr

Wörter, die eine spezifische Bedeutung aufweisen: z.B. Bäckerei ist auch süßes Kleingebäck, man kann nicht nur Finger spreizen, sondern im Garten werden auch die fruchtschweren Äste der Obstbäume gespreizt.

Manche Wörter, die früher nur auf Österreich beschränkt waren, wurden erst dann von dem Binnendeutschen übernommen. Im Laufe der Zeit haben sich diese Ausdrücke so stark eingewurzelt, daß die Grenze zwischen dem Österreichischen und dem Deutschen schwer zu erkennen ist. Wahrscheinlich deshalb werden viele deutsche Wörter in den großen deutschen Wörterbüchern nicht als „österr." gekennzeichnet, also ohne regionale Begrenzung.

\section{Analyse der Austriazismen}

Als Grundlage zur Analyse der österreichischen Besonderheiten dienten mir die österreichischen Zeitungen, vor allem die „Neue Kronen Zeitung”, die österreichischen Wörterbücher, die Arbeiten der Sprachwissenschaftler Hildegard Rizzo-Baur, Peter Wiesinger, Jakob Ebner, Ulrich Ammon aber vor allem die gesprochene Sprache. Die analysierten Belege kommen aus dem Bereich „Essen” und wurden in fünf verschiedenen Wörterbüchern nachgeschlagen.

Auffallend viele Abweichungen begegnen uns in der Küchensprache. Zum Teil handelt es sich dabei um Bezeichnungen typisch österreichischer Gerichte, welche dem Speisezettel in Binnendeutschland fremd und deren Namen daher dort ungeläufig oder unbekannt sind; andere Speisen dagegen sind zwar Gemeingut im gesamten deutschen Sprachgebiet, doch ist die Bezeichnung landschaftlich verschieden (Rizzo-Baur 1962:37).

In der Benennung der einzelnen Teilstücke übertrifft Österreich das Binnendeutsche an Originalität und Vielfältigkeit. So entspricht das Selchkarree (das; -s/-s: „geräuchertes Fleisch"; DTb²) einem Kasseler Rippenspeer, das Kaiserfleisch einem geräucherten Schweinebauch (DTb). Das Rippstück verkauft man hier als Schweins- oder Kalbskarre (zu frz. carrè). Eine Rindfleischsorte wird als Schulter- oder Hüferscherzel verkauft (Scherzel, das; -s/-: a) End-

${ }^{2}$ DTb: Duden Taschenwörterbücher. Wie sagt man in Osterreich? 1980. 
stück des Brotleibes, auch bayr. b) „Fleischteil beim Rind zwischen den Hinterbeinen und den Hüften"; DTb); Scherzel steht wohl für Schörzel und stammt aus it. scorza (Rindenstück).

Die „„ßbare Lunge” der Tiere heißt in Österreich das Beuschel (das; -s: a) Speise aus Tierinnereien, bes. Herz und Lunge; b) /derb/ Lunge; DTb). Dem binnendeutschen Rauchfleisch wiederum entspricht das Geselchte oder Gselchte (das; -n: geräuchertes Schweinefleisch, auch bayr., DTb). Aus der Mundart stammt der Name Surfleisch (das; -es: Pökelfleisch; DTb). Frikadellen finden wir auf der ôsterreichischen Speisekarte als Fleischlaibchen oder Fleischlaiberl (ostösterr. Fleischlaberl, das; -s/-n: für binnendt. Bulette/bes. Berlin/; DTb).

Gebratenes Hähnchen wird zu Backhendl (das; -s/-n; ÖWb ${ }^{3}, \mathrm{DTb}$ ). Leberkäse (der; -s: entspricht in Österreich, mit Ausnahme von Tirol, ungefähr dem in Deutschland üblichen Fleischkäse, wird also ohne Leber hergestellt; DTb, Leberkäs: eine „Wurstsorte"; ÖWb) der in Österreich und in Süddeutschland besonders beliebt ist, ist ein gebackenes oder gekochtes Fleischgericht, das meistens (in vorwiegendem Teil Österreichs) weder aus Leber noch aus Käse hergestellt wird (es gibt aber auch Leberkäse extra mit Käse). Es ist ein Gericht aus sehr fein gehacktem Rind- und Schweinefleisch mit Gewürzen (vgl. Wahrig 1996:1002). $\mathrm{Zu}$ den Fleischspezialitäten gehört auch die Blutwurst, die hier Blunze(n) (ugs., die; -/-n; DTb) genannt wird.

Das Bauchfett des Schweins kennt der Österreicher als Filz (der; -es/-e: bedeutet österr. auch Schweinespeck, der zu Schmalz ausgelassen wird; DTb). Rückstände, die beim Ausbraten des Schweinespecks entstehen, werden als Grammeln (die -/-n: „Griebe, Speckgriebe”, auch bayr.; DTb) bezeichnet. Das binnendeutsche Eisbein wird hier durch Stelze (die; -/-n: verkürzte Form für „Schweinsstelze"; DTb) ersetzt.

Den unteren Teil des Beines, nicht nur beim Schwein nennen die Österreicher Hax(e)l (das; -s/-n; DTb). Haxeln werden auch in der Umgangssprache die Beine des Menschen genannt.

Zur Bereitung einer Speise, die man mit Fleisch füllt, brauchen die Österreicher Faschiertes (das; -n: Hackfleisch oder Speisen aus Hackfleisch, bes. faschierte Laibchen, faschierter Braten; DTb).

Im Österreichischen finden wir auch z.B. auf einer Speisekarte eine Fischart, die für einen Deutschen fremd klingt. Fogosch (der; -es/-e: eine Fischart „Schill, Zander”; DTb) entstammt dem Ungarischen und entspricht dem deutschen Wort Zander, ung. fogas = Zander (Ammon 1995:80).

Die Bezeichnungen für Obst und Gemüse decken sich ebenfalls nicht immer mit den entsprechenden binnendeutschen. Neben der gemeindeutschen Vollform Hollunder ist hier die mundartliche Kurzform Holler (der; -s/-; österr.

${ }^{3}$ Öwb: Ōsterreichisches Wörterbuch, 1979. 
und süddt.; DTb). Ebenso gibt es: Hollerbeere, Hollerblüte, Hollerbusch oder Hollerschnaps. Neben der Heidelbeere finden wir in Österreich die Schwarzbeere (die; -/-n: österr. und bayr.; DTb). Im östlichen Teil Österreichs wird oft in der Mundart statt Stachelbeere das Wort Agrasel (das; -s/-n; DTb) verwendet.

Der deutschen Zwetsche - einer Sorte Pflaumen, entspricht die Zwetschke (die; -/-n: österr. nur so gebrauchte Form für binnendt. Zwetsche, schweiz. und süddt. Zwetschge). Das Wort Pflaume trifft man kaum in Österreich. Und eine Marmelade, die aus Zwetschken gemacht wird, heißt hier Powidl (der; -s, bes. im Osten Österreichs: Pflaumennuß; DTb). Der Powidl wird zu allerlei z.B. den beliebten Powidlknödeln verarbeitet. Dieses Wort stammt aus dem tschech. powidla = Mus, Obstmus.

Die binnendeutsche Aprikose ist in Österreich als Marille (die; -/-n:österr.) bekannt. Auch solche Zusammensetzungen Marillenlikör, Marillenkompott, Marillenschnaps, Marillenmarmelade, und Marillensoße oder Marillenknödel. Das Wort ist aus einer Verschmelzung des italienischen armenilo, armelino (Aprikose) mit Amarelle, Morelle (it. amarelia, amarena, amresca entstanden; vgl. Kretschmer 1969:135). Ein weiteres Beispiel, das hier angeführt werden soll ist die Ribisel (die; -/-n: österr., außer Vorarlberg; DTb), die der Johannisbeere entspricht. Ursprünglich war die Bezeichnug Ribisel nur für die rote, heute ist sie auch für die schwarze Sorte. Ribisel stammen aus dem italienischlateinischen Wort ribes (Ammon 1995:179).

Die Kartoffel ist in Österreich unter dem Namen Erdapfel bekannt (der; -s/-äpfel; DTb). An Stelle des binnendeutschen Rosenkohls finden wir hier Sprossenkohl (der; -s: österr.; DTb) oder Kohlsprossen (die; -/-n). Die aus Amerika stammende Pflanze - Mais wird hier Kukuruz (der; -; DTb) genannt, aus dem kroat. $k u k u r u z=$ Mais (Ammon 1995:180; vgl. Ebner 1988:169).

Die Bezeichnung Tomate gebraucht der Österreicher vor allem in den $\mathrm{Zu}-$ sammensetzungen z.B. Tomatenmark. Statt Tomate schreibt er oft Paradeiser (der; -s/-: österr., außer Tirol und Voarlberg; DTb). Der Paradeiser für Tomate ist eine Lehnübersetzung von pomodoro (Ammon 1995:179). Melanzani (die, Pl.: österr. Form für binnendt. Melanzane; Auberginen; DTb) hat nur eine andere Endung als das italienische melanzana (ebda.). Blumenkohl heißt hier Karfiol (der; -s: österr. und süddt.; DTb). Der Karfiol für Blumenkohl, durch Zusammenziehung des gleichbedeutenden italienischen cavalfiore (Ammon 1995:179) entstanden, war bereits vor einigen Jahren in Deutschland eingedrungen, wurde dort aber durch die Übersetzung verdrängt. In Österreich dagegen wurde der romanische Ausdruck bis heute beibehalten. Dem binnendeutschen Wort Meerettich entspricht das Wort Kren (der; -s: österr. und süddt.; DTb). Dieses Wort weist slawischen Ursprung auf: russ. хрен, tschech. křen (Ammon 1995:180). Der Rettich wird hier umgangssprachlich Radi (der; -s/-: auch bayr.; DTb) genannt. Der Pfefferoni (der, -/-, oder selten Pfefferone : „kleine, längliche sehr 
scharfe Paprikasorte"; DTb) ist in Anlehnung an Pfeffer aus dem it. pepperone entstanden. Der binnendeutschen Bezeichnung Marone (eßbare Frucht der Kastanie) entspricht die Form Maroni (die; -/-: österr. und süddt.; DTb).

Den grünen Bohnen entsprechen wiederum Fisolen (die; -/-n; DTb). Dem Pilz wird sehr oft der Schwamm (der; -(e)s /Schwämme; DTb) oder das Schwammerl (ugs.,das; -/-n: auch bayr.; DTb) vorgezogen. Die Verkleinerung besagt aber nicht, daß es sich um einen kleinen Pilz handelt. Der Pfifferling ist hier als Eierschwammerl bekannt.

Ein Kompott aus Holler oder aus Zwetschken wird als Röster (der; -s/-: österr.; DTb) bezeichnet. Ein mit Sodawasser verdünnter Wein heißt in Österreich ein Gespritzter (der; -/-n: auch bayr.; DTb). Bei Heurigen (der; -n/-n: Lokal bestimmter Art, in dem neuer Wein / mit besonderer behördlicher Bewilligung / aus den eigenen Weinbergen des Besitzers ausgeschenkt wird; DTb) finden wir oft auf der Getränkeliste den Sturm (der; -(e)s: ,in Gärung übergangener Traubensaft"; DTb), - das erste Stadium der Weinproduktion. Der Most, ein alkoholischer Obstsaft, das zweite Stadium der Weinproduktion (der; -es: auch süddt. und schweiz.; DTb), ist in dieser Form vor allem in Österreich zu finden.

Kranewitter (der; -s; DTb) bezeichnet in Österreich einen Wacholderschnaps. Die Bezeichnung Kracherl (ugs., das; -/-n: (veraltend) Limonade; DTb) ahmt das Geräusch nach, das infolge des Kohlensäuregehaltes beim Öffnen der Flasche verursacht wird und deshalb entspricht der Brausenlimonade.

Wenn wir in einem österreichischen Lokal einen Kaffee trinken möchten, ist es nicht immer leicht den richtigen $\mathrm{zu}$ bestellen.

Der Mocca (der; -s/-s: in Österr. meist gebräuchliche Form neben Mokka; DTb) ist ein besonders starker schwarzer Kaffe. Die Melange (die; -/-n: bezeichnet eine Zubereitungsart von Kaffee; DTb) ist ein Kaffee mit heißer Milch und viel Milchschaum. Der Kapuziner (der; -s/-: bezeichnet in Österr. auch eine Zubereitungsart von Kaffee; DTb) bedeutet schwarzen Kaffee mit einem Schuß Milch, der Einspänner (der; -s/-: bedeutet in Österr. auch eine Zubereitungsart von Kaffee im Kaffeehaus; DTb) ist ein Mocca mit Schlagobers, der im Glas serviert wird und der Franziskaner ist eine Melange mit Schlagobers und Schokostreuseln. Dem Französischen entstammt die Bezeichnung für ein Milchgetränk - Frappeè (das; -s/-s: „eisgekühltes Getränk aus Milch, vermischt mit zerkleinerten Früchten z. B. Bananen, Erdbeeren"; DTb).

Quark kennt der Österreicher nicht. Dieses Milchprodukt heißt hier Topfen (der; -s: österr. und bayr.; DTb). Ähnlich dem Quark klingt das Quargel (der; -s/-: „kleiner, runder, stark riechender Käse; Harzer Käse, Ölmützer Stinkkăse”; DTb). Sahne, die in Österreich ungebräuchlich ist, heißt Obers (das; -: besonders ostösterr. und bayr. für süße Sahne; DTb).

Zur Bereitung eines Hefeteigs brauchen die Österreicher Germ (die; -/-: „Backhefe"; DTb). Diese Bezeichnung wird auch in Süddeutschland gebraucht, dort aber immer als Maskulinum. 
Einer binnendeutschen Fleischbrühe entspricht die österreichische Suppe, genauer Rindsuppe genannt. Wenn wir in einem Lokal z.B. eine Backerbsensuppe bestellen, bekommen wir eine Rindfleischbrühe mit einer Suppeneinlage aus Backteig in Form von Erbsen. Die in Wien bekannte Panaden- oder Panad/e/suppe (die; -/-n: Rindsuppe mit einer Einlage aus Weißbrotschnitten, auch süddt.; DTb) ist aus der französischen panade entstanden. Anstatt der in Süddeutschland bereiteten Flädelsuppe (Suppe mit einer Einlage aus dünnen, in Streifen geschnittenen Pfannkuchen) läßt sich der Österreicher eine Frittatensuppe schmecken (zu it. frittata - Eierkuchen).

Die Bezeichnung Semmelbrösel (die /Plural/: bes. österr. und bayr.; DTb) wird für das binnendeutsche Paniermehl oder Semmelmehl gebraucht. Diese Bezeichnung für Mehl aus geriebenen, harten Brötchen ist nach „Handwörterbuch der deutschen Gegenwartssprache" (1984) von G. Kempcke nicht nur für Österreich spezifisch, sondern wird auch in anderen Regionen Deutschlands gebraucht.

Das Piment nennen die Österreicher Neugewürz (das; -es; österr.; DTb). Rühreier sind hier unter dem Namen Eierspeise (die: -/-n: österr.; DTb) bekannt. An Stelle der binnendeutschen Bezeichnung Klo $\beta$ verwendet der Österreicher nur Knödel (der; -s/-: österr. und süddt.; DTb).

Das Aussehen und Beschaffenheit rohen und gekochten Eiweißes unterscheidet der Östrreicher auch durch die Benennung Eiklar (das; -(e)s/-(e): „,weißer Teil im Ei”; DTb). Eiklar bedeutet „flüssiges Eiweiß”, es wird auch einfach Klar genannt.

Das Wort Mehlspeise erfullt in Österreich die Funktion des breiteren Oberbegriffs. „Dieser Begriff ist in Österreich semantisch stark besetzt und hat zwei Bedeutungen: 1. 'jede Speise aus Mehl als Gegensatz zu Fleischspeise' (diese Bedeutung ist Gemeindeutsch) 2. 'eine süße oder salzige Speise, die aus Mehl, Eiern zubereitet wird', z.B. Topfenpalatschinken oder Germknödel. Die Verwendung geht oft soweit, daß das Wort für Speisen ohne Mehl verwendet werden kann. 3. Binnendeutsch 'Kuchen'." (Ebner 1988:123). Dieses Wort wird auch im Sinne von Nachtisch verwendet.

$\mathrm{Zu}$ den typischen Mehlspeisen in Österreich gehören u.a.: die Palatschinke (die; -n/-, /meist Plural/: „dünner Eierkuchen, der zusammengerollt und mit Marmelade o.ä. gefüllt wird”; DTb), die Schaumrolle (die; -/-n: „Rolle aus Blätterteig, die mit Schlagobers/Schlagsahne gefüllt ist"; DTb), das Vanillekipferl (das; -s/-n: „süßes Nuß- oder Mandelgebäck in Form von sehr kleinen Hörnchen, mit Vanillezucker bestreut”; DTb), der Krapfen (der; -s/-: „eine Mehlspeise aus Hefeteig, die im Fett gebacken wird", auch süddt. und bayr.; DTb) entspricht dem in Deutschland und in der Schweiz gebrauchten Begriff Berliner oder Berliner Pfannkuchen.

Das Wort Palatschinke ,stammt ursprünglich aus dem Lateinischen (lat. placenta $=$ flacher Kuchen), von wo es zunächst ins Rumänische gelangt. Von 
dort wird es ins Ungarische (palascinta) übernommen, und erst von da aus findet es schließlich seinen Weg ins österreichische Deutsch" (Ammon 1995:179). Unter zahlreichen österreichischen Mehlspeisen finden wir auch folgende tschechische Namen: Buchteln (die; -/-n, meist Plural: „ein Gebäck aus Hefeteig, oft mit Marmelade o.ä. gefüllt"; DTb), tschech. buchticky = Dampfnudel (ebda.). Golatsche/Kolatsche (die; -/-n: „kleiner, gefüllter Hefekuchen, urspr. rund, jetzt meist viereckig, bei dem alle vier Ecken einer größeren Küchenfläche nach innen gebogen wurden", bayr. Tascherl; DTb), tschech. kolač (ebda.). Skubanki/Skuwanki/Skubanken (die; Plural, bes. Wien: „Speise aus Kartoffeln, Mehl, Butter, die in Form von Nockerln ausgostochen, mit zerlassener Butter übergossen und mit Mohn bestreut wird"; DTb), „vielleicht zu tschech. skyva = Brotschnitte (Rizzo-Baur 1962:85). Löffelbiskuits (Biskuits in Achterform) führen in Österreich den Namen Biskotten (die; -/-n: „Biskuit in länglicher Form“; DTb) zu it. biscotti (Kleingebäck).

Das Wort Gebäck ist in Österreich ein Oberbegriff für Semmeln (die; -/-n: Brötchen; W), Kipfel/Kipferl (das; -s/-n: „kleines, gebogenes Weißbrotgebäck, auch bayr.: DTb), Weckerl (das; -s/-n: „kleines, längliches Gebäck, auch bayr.; DTb) usw., im Binnendeutschen dagegen versteht man darunter ein „feines, süßes Backwerk in geformten, festen Einzelstücken" (vgl. ebda., S. 112). Großer Beliebtheit erfreut sich in Österreich das Wort Zuckerl (das: -s/-n; DTb), das statt der Bezeichnung Bonbon verwendet wird.

Tabelle 1: Wortliste einiger ausgewählten österreichischen Besonderheiten in den Wörterbüchern.

\begin{tabular}{|l|c|c|c|c|}
\hline \multicolumn{1}{|c|}{ Wort } & DTb & Ōwb & W & GDP \\
\hline Agrasel & + & + & & \\
\hline Backerbsen & + & + & & \\
\hline Biskotte & + & + & + & $+^{\mathbf{a}}$ \\
\hline Blunzen & + & + & & \\
\hline Eierschwamm/erl/ & + & + & + & \\
\hline Eierspeise & + & + & + & + \\
\hline Eiklar & + & + & + & $+^{\mathbf{b}}$ \\
\hline Einspanner (Kaffee) & + & + & + & \\
\hline Erdapfel & + & + & + & + \\
\hline Faschiertes & + & + & & \\
\hline Filz (Speck) & + & + & & \\
\hline Fleischleibchen & + & + & & \\
\hline Fogosch & + & + & & \\
\hline Frittate/n(suppe) & + & + & + & \\
\hline Geselchte & + & + & + & + \\
\hline Golatsche & + & + & + & + \\
\hline Grammeln & + & + & + & + \\
\hline Ha/x/el & + & + & & + \\
\hline
\end{tabular}




\begin{tabular}{|c|c|c|c|c|}
\hline Wort & DTb & Öwb & $\mathbf{W}$ & GDP \\
\hline Hendl & + & + & + & $+^{\mathrm{c}}$ \\
\hline Holler & + & + & + & + \\
\hline Innereien & & + & & + \\
\hline Kaiserfleisch & + & + & & \\
\hline Kapuziner & + & + & & + \\
\hline Karfiol & + & + & + & + \\
\hline Kipfe/r/l & + & + & + & + \\
\hline Kohlsprossen & + & + & & \\
\hline Kracherl (veraltet) & + & + & + & $+^{d}$ \\
\hline Kren & + & + & + & + \\
\hline Leberkäse & + & + & + & + \\
\hline Lungenbraten & + & + & & \\
\hline Marille & + & + & + & + \\
\hline Maroni & + & + & & \\
\hline Mehlspeise & + & + & + & \\
\hline Melange & + & + & + & + \\
\hline Melanzani & + & + & + & \\
\hline Most & + & $t$ & + & + \\
\hline Neugewürz & + & + & + & \\
\hline Palatschinke & + & + & + & \\
\hline Panadel(suppe) & + & + & + & + \\
\hline Paradeiser & + & + & + & \\
\hline Powidl & + & + & + & \\
\hline Quargel & + & + & + & $t^{e}$ \\
\hline Ribis/e/l & + & + & + & + \\
\hline Röster & + & + & + & \\
\hline Scherzel (Fleischteil) & + & + & & \\
\hline Selchkarree & + & + & & \\
\hline Semmelbrösel & + & + & + & \\
\hline Skubanki & + & + & & \\
\hline Stelze (Eisbein) & + & + & & \\
\hline Surfleisch & + & + & & \\
\hline Weckerl & + & + & & \\
\hline Zeller & + & + & & \\
\hline Zuckerl & + & + & + & + \\
\hline Zwetschke & + & + & $t^{t}$ & \\
\hline
\end{tabular}

a es fehlt hier an Bezeichnung ,austr. ${ }^{\text {" }}=$ „osterreichisch ${ }^{\text {" }, ~}{ }^{\mathrm{b}}$ im Nachtrag von T. Kachlak, ${ }^{\mathrm{c}}$ ebenda,

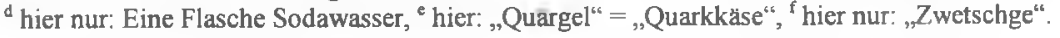

DTb - Duden Taschenwörterbuch. Wie sagt man in Österreich?, 1980.

OWb - Ósterreichisches Worterbuch, 1979.

W - Wahrig. Deutsches Worterbuch, 1996.

GDP - Großworterbuch Deutsch-Polnisch, 1981.

Ich habe versucht die Spezifik der österreichischen Sprache darzustellen anhand der Besonderheiten (vor allem des Wortschatzes), die im alltäglichen Leben am 
häufigsten zu lesen oder zu hören sind und deren Unkenntniss zu einer Verständnisstörung führen könnte. Die Liste der Austriazismen, die dieser Artikel umfasst, enthält nicht alle Begriffe, da die Vokabeln nur aus dem von mir ausgewählten Lebensbereich stammen.

Einige der von mir in vier verschiedenen Wörterbüchern (,Wie sagt man in Österreich?", Wörterbuch der österreichischen Besonderheiten, Wahrig. Deutsches Wörterbuch, Österreichisches Wörterbuch. und Großwörterbuch Deutsch-Polnisch.) nachgeschlagenen Wörter sind nicht nur auf Österreich begrenzt, sondern auch für den Süddeutschen Raum und manchmal auch für die Schweiz spezifisch. Nicht alle von den nachgeschlagenen Vokabeln sind in den von mir getesteten Wörterbüchern präsent. Die meisten Begriffe findet man in dem Wörterbuch der österreichischen Besonderheiten und in dem österreichischen Wörterbuch, seltener in dem Deutsch-Polnischen Wörterbuch und in dem Deutschen Wörterbuch von G. Wahrig (siehe Tabelle 1.) und in den zwei letzten, von mir obengenannten wird nicht immer auf die Spezifik der Wörter, die im Binnendeutschen vorkommen, in Österreich aber eine andere Bedeutung aufweisen, verwiesen.

\section{LITERATUR}

Ammon, U. (1995): Die deutsche Sprache in Deutschland, Österreich und der Schweiz. Das Problem der Nationalen Varietaten. Berlin/New York.

Benedikt, E., Hornung, M., Pacolt, E. (1979): Österreichisches Wörterbuch. Wien.

Bürkle, M. (1995): Zur Aussprache des österreichischen Standarddeutschen. Die unbetonten Silben. Frankfurt/Main.

Drosdowski, G. (Hg.) (1976): Duden. Das graße Wörterbuch der deutschen Sprache. Mannheim.

Ebner, J. (1969): Duden. Wie sagt man in Osterreich? Wörterbuch der österreichischen Besonderheiten. Mannheim/Wien.

Fenske, H. (1973): Schweizerische und österreichische Besonderheiten in deutschen Wörterbüchern. Mannheim.

Fleischer, Wolfgang (1983): Kleine Enzyklopädie „Deutsche Sprache “. Leipzig.

Földes, C. (1996): Rezension zu Ulrich Ammon: Die deutsche Sprache in Deutschland, Österreich und der Schweiz. Das Problem der nationalen Varietāten. In: Muttersprache 1/97, S. 86-89.

Kątny, A. (1993): „,Falsche Freunde ${ }^{\text {sc }}$ in den deutsch-polnischen Beziehungen. In: A. Kątny (Hg.) Beiträge zur Sprachwissenschaft, Sozio- und Psycholinguistik. Probleme des Deutschen als Mutter-, Fremd- und Zweitsprache. Rzeszów. S. 59-61.

Lewandowski, Th. (1990): Linguistisches Wörterbuch, 5. überarbeitete Auflage; Heidelberg/Wiesbaden.

Neue Kronen Zeitung, Tageszeitung (1997/1998/1999). Wien.

Pfalz, Anton (1937): Die Mundarten des Norddonauraumes. Leipzig.

Piprek, J.; Ippoldt J. (1981): Großwörterbuch Deutsch-Polnisch. Warszawa.

Rizzo-Baur, H. (1962): Die Besonderheiten der deutschen Schriftsprache in Österreich und in Sildtirol. H. Moser (Hg.). Mannheim. 
Siebs, T. (1969): Deutsche Aussprache. 19 Auflage. Berlin.

Steinhauser, W. (1961): Zur bairisch-osterreichischen Lautgeschichte. In: Zeitschrift fur Mundartforschung 28, S. 166-183.

Takahashi, H. (1996): Ober Michaels Burkle: Zur Aussprache des osterreichischen Standarddeutschen. Die unbetonten Silben. In: Soziolinguistica 10/1996, S. 151-152.

Wehle, P. (1980): Sprechen Sie Wienerisch? Wien.

Wiesinger, P. (1980): Zum Wortschatz im ōsterreichischen Wörterbuch. In: Österreich in Geschichte und Literatur, 24. Jg. 1980/7. Sonderpublikation des Institutes fur Osterreichkunde (Beiheft).

Wiesinger, P. (Hg.) (1988): Das ठsterreichische Deutsch. In: Schriften zur deutschen Sprache in Osterreich. Band 12. Wien-Koln-Graz.

Wiesinger, Peter (1988): Die deutsche Sprache in Osterreich. Eine Einfihrung. In: Wiesinger, P. (Hrsg.).

Wintersberger, Astrid (1995): Osterreichisch-Deutsches Wörterbuch. Wien. 